\title{
Geometry-based channel modelling of MIMO channels in comparison with channel sounder measurements
}

\author{
G. Del Galdo, M. Haardt, and C. Schneider \\ Communications Research Laboratory, Ilmenau University of Technology, P.O. Box 100565, 98684 Ilmenau, Germany
}

\begin{abstract}
In this paper we propose a flexible geometrybased propagation model for wireless communications developed at Ilmenau University of Technology. The IlmProp comprises a geometrical representation of the environment surrounding the experiment and a precise representation of the transmitting and receiving antennas. The IlmProp is capable of simulating Multi-User MIMO scenarios and includes a complete collection of tools to analyze the synthetic channels. In order to assess the potentials as well as the limits of our channel simulator we reconstruct the scenario encountered in a recent measurement campaign at Ilmenau University of Technology leading to synthetic data sets similar to the ones actually measured. The measurements have been collected with the RUSK MIMO multi-dimensional channel sounder. From the comparisons of the two channel matrices it is possible to derive useful information to improve the model itself and to better understand the physical origins of small-scale fading. In particular the effects of the different parameters on the synthetic channel have been studied in order to assess the sensibility of the model. This analysis shows that the correct positioning of a small number of scatterers is enough to achieve frequency selectiveness as well as specific traits of the channel statistics. The size of the scattering clusters, the number of scatterers per cluster, and the Rician $K$-factor can be modified in order to tune the channel statistics at will. To obtain higher levels of time variance, moving scatterers or time dependent reflection coefficients must be introduced.
\end{abstract}

\section{Introduction}

The high complexity of the propagation phenomenon sets a vast challenge in the effort of modelling the wireless channel. The most accurate approach would be to solve the Maxwell equations. However the computational effort required would be prohibitive, especially if we considered multiple antennas

Correspondence to: G. Del Galdo

(giovanni.delgaldo@tu-ilmenau.de) at both ends of the link, i.e. a MIMO system (Multiple Input Multiple Output). In fact, if $M_{R}$ and $M_{T}$ are the number of antennas at the receiver and at the transmitter, respectively, the number of channels to be calculated grows to $M_{R} \cdot M_{T}$. Furthermore a precise physical and geometrical description of the objects would be necessary.

In order to reduce the complexity of the model the so called GTD (Geometric Theory of Diffraction) can be employed (Correia, 2001). This approach postulates the existence of direct, diffracted and reflected rays only. As the wavelength $\lambda$ approaches zero, this approximation becomes increasingly accurate.

With a ray-tracing engine and a full 3D description of the environment it is then possible to calculate all possible rays linking the antennas. However with a GTD approach a complete geometrical representation of the scenario is still required. A further approximation is to simplify the geometry surrounding the antennas with a discrete number of reflectors, called scatterers; when only direct and reflected rays are considered the computational complexity drops significantly. In this paper we present the IlmProp, a simplified model which generates channels which mimic most characteristics of measured channels. To this aim it is extremely important to determine which features of the synthetic channel are affected by the different parameters present in the model. A relatively low computational complexity allows us to generate channels with all possible combinations of values for the parameters. Then we analyze the data by finding strong traits which link a particular parameter to a particular feature. At the same time we are able to assess whether a parameter has no effect on a specific feature.

This extensive search and its results are reported in Sect. 3. Then we modeled the scenario found in one of the measurement campaigns undertaken at Ilmenau University of Technology. Section 4 reports the direct comparison between the synthetic and measured channels which led to a better understanding on the origins of the fast and slow fading processes. Section 5 deals in particular with the comparison of the phase responses. Lastly, Sect. 6 concludes this paper. 


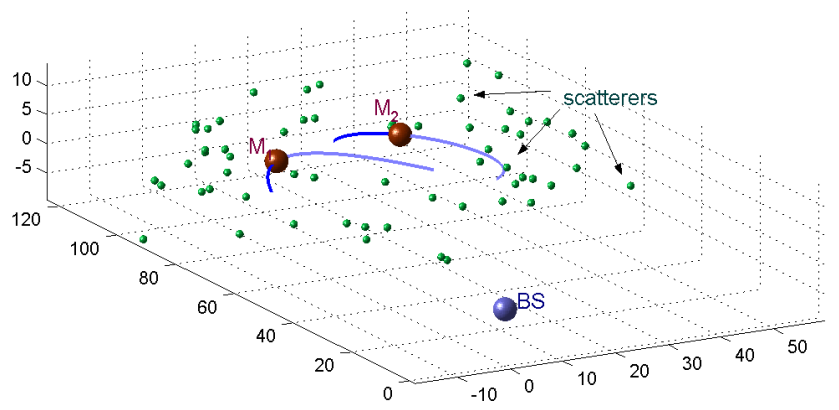

Fig. 1. In this example 2 Mobiles (M's) surrounded by scatterers move in the proximity of one fixed Base Station (BS).

\section{The IlmProp: a geometry-based channel model}

The IlmProp developed at TU Ilmenau relies entirely on a 3D geometric representation and has been designed to simulate a Multi-User-MIMO scenario. It has been simplified to single-bounce reflections in order to reduce the computational complexity. Clusters of scatterers can be arbitrarily positioned in the 3D reconstruction of the environment. Each scatterer corresponds to a single ray and is characterized by a complex coefficient which, for simplicity, is independent of the Direction of Arrival (DoA) and the Direction of Departure (DoD); but may vary in time with an arbitrary law. The coefficient determines the phase shift and power attenuation introduced by the scatterer.

The receiver thus receives a number of rays equal to the number of scatterers plus the Line Of Sight (LOS), which links the transmitter to the receiver directly. Figure 1 shows a simple example of this modelling approach. Such a simplified model still requires a fairly high computational complexity because the total number of rays to be computed is: $M_{R} \cdot M_{T} \cdot N_{\text {scatt }}$.

The advantage of a geometry-based model with respect to a stochastic one is indeed the possibility to easily generate a frequency selective time variant channel. In fact the frequency selectiveness is caused by the multi-path effect while the time variance is set off by the movement of the antennas and of the scatterers as well as the varying scattering coefficients with respect to time. In other words it well models a typical frequency selective time variant radio channel. In order to characterize such a channel we need to describe it in one of the four possible two-dimensional domains named the Bello domains. (Bello, 1963). Either $\{t, f\},\{t, \tau\},\left\{f_{D}, f\right\}$ or $\left\{f_{D}, \tau\right\}$ where $t, f_{D}, f$, and $\tau$ denote time, Doppler frequency, frequency, and delay time, respectively. The most convenient domain in which to compute the channel is certainly $\{t, f\}$. Having a geometrical representation of all objects in time makes the choice of $t$ over $f_{D}$ quite obvious. The choice of $f$ over $\tau$ requires more explanation. Thanks to the geometrical representation it is simple to calculate the path lengths for each ray present in the scenario. Assuming that the velocity of light, $c$, is equal to the velocity of all rays, we can calculate the delay time associated with every ray, i.e. the time needed to reach the receiver. The delay times found will not necessarily match the sampling grid chosen. For this

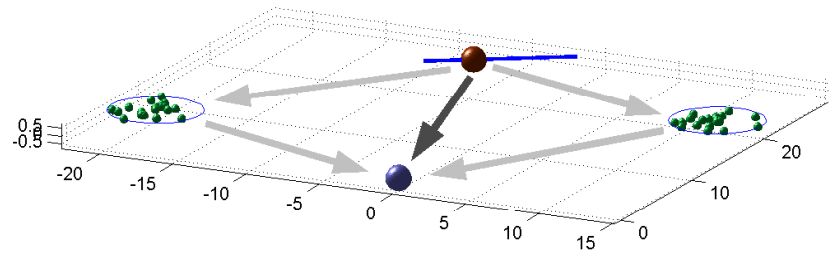

Fig. 2. The geometrical representation of the model $\mathcal{M}_{\alpha}$. Two scattering clusters and a strong LOS are visible. The transmitter (at the top) moves on a linear path.

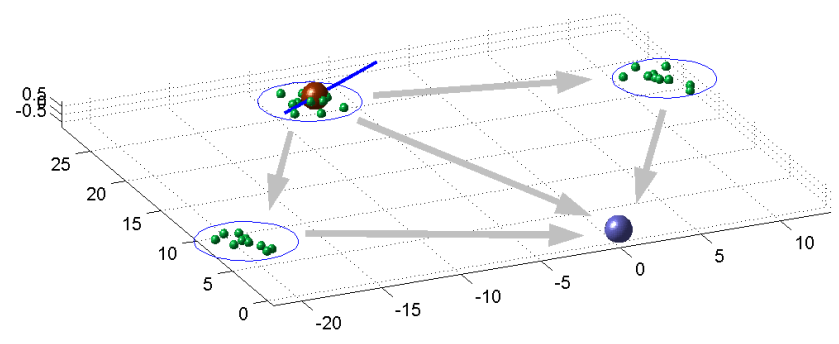

Fig. 3. The geometrical representation of the model $\mathcal{M}_{\beta}$. The model is characterized by three scattering clusters; one of them surrounds the transmitter.

reason more processing is required. On the contrary, in the frequency domain we can calculate the channel response for the specific frequency bins that we are interested in. The choice of a specific Bello domain is at the end a matter of convenience since it is possible to derive the other representations via Fourier transforms. For instance, having $H(f, t)$, i.e. the channel expressed in delay time and time, we can calculate $h(\tau, t)$, the channel in frequency and time, as:

$h(\tau, t)=\int H(f, t) \mathrm{e}^{j 2 \pi f \tau} d f$.

The amplitude and phase of each ray are calculated separately at the receiver. The phase of the $i$-th ray at a certain time snapshot $t_{o}$ and frequency $f_{o}$ are calculated as follows:

$\varphi_{i}\left(t_{o}, f_{o}\right)=L\left(\mathrm{e}^{-j 2 \pi \frac{f_{o}}{c} L_{i}}\right)+\theta_{i}$,

where $L_{i}$ is the ray's path-length and $\theta_{i}$ is the phase shift introduced by the scatterer. The amplitude depends simply on the scattering coefficient, antenna gain, and path-loss (Van Trees, 2002).

\section{An exhaustive investigation}

The first step in understanding how sensible the model is to the different parameters and which features of the synthetic channel are affected, is to model some typical scenarios and to generate channels with all possible values for the parameters. We implemented two models, to which we will refer as $\mathcal{M}_{\alpha}$ and $\mathcal{M}_{\beta}$, seen in Figs. 2 and 3. $\mathcal{M}_{\alpha}$ models a typical scenario in which the areas around the transmitter and the receiver are free of scatterers while some are present in the 
middle of the link. $\mathcal{M}_{\beta}$, on the other hand, models a scattering cluster in the proximity of the transmitter. We generated each channel for the following sampling grid:

- A bandwidth $B$ of $400 \mathrm{MHz}$ at a center frequency of 1 $\mathrm{GHz}$ sampled with a $\Delta f=1.3 \mathrm{MHz}$ for a total of 301 samples.

- A total of 200 time snapshots were taken, one every 10 $\mathrm{ms}$ for a total duration of $2 \mathrm{~s}$.

The choices of these parameters determine the sampling grids in the coupled domains. In fact if the complex baseband signal is sampled in the range $\left[-\frac{B}{2}, \frac{B}{2}\right]$, then $\Delta \tau$, the resolution in delay time, is equal to $\frac{1}{B}$. In the same way the bandwidth in the Doppler frequency, $2 \cdot f_{D, \max }$ can be calculated from the resolution in time $\Delta t$ as:

$f_{D, \max }=\frac{1}{2 \cdot \Delta t}$.

- The resolution in delay time is $\Delta \tau=2.5 \mathrm{~ns}$, which corresponds to a distance of less than a meter $(75 \mathrm{~cm})$. We can successfully resolve paths (i.e. without aliasing), which are up to 220 meters long.

- Doppler shifts up to $f_{D, \max }= \pm 50 \mathrm{~Hz}$ are visible without aliasing.

The transmitter moves with a uniform velocity of about 5 $\mathrm{m} / \mathrm{s}$. Both transmitter and receiver employ a ULA (Uniform Linear Array) with $M_{R}=M_{T}=4$ antennas spaced by $\frac{\lambda}{2}$.

After several experiments with different geometries and different configurations we narrowed down our extensive search to three dominant parameters:

- Cluster size

- Number of scatterers per cluster

- Rician $K$-factor

A fourth dominant parameter is certainly the modelling of the reflection coefficients, and more importantly their trend in time. This leaves, however, too many degrees of freedom since we could implement any arbitrary function to describe it in time. For this reason we left this important parameter to future and more specific investigations. In our simulations the reflection coefficients are kept constant along all dimensions, i.e. frequency and time. The Rician $K$-factor is defined as

$K=\frac{P_{\mathrm{LOS}}}{P_{\text {scatt }}}$,

i.e. the power attributed to the Line Of Sight divided by the power of the scatterers. Clearly it is a function of the pathlosses which on their side are determined by the geometry, the transmit power, and the reflection coefficients. We scale the amplitudes of the reflection coefficients to obtain a desired $K$-factor.

We then generate many channels with different values for these three parameters and then analyze the data to find any strong dependency.

\section{$3.1 K$-factor}

The $K$-factor affects several features of the channel. Firstly it influences its variance with respect to all four domains. It is thus very interesting to assess this information quantitatively computing the RMS delay spread $\tau_{\mathrm{RMS}}$, the coherence bandwidth $(\Delta f)_{\mathrm{c}}$, the RMS Doppler spread $f_{\mathrm{D}, \mathrm{RMS}}$ and the coherence time $(\Delta t)_{\mathrm{c}}$.

The RMS delay spread of the channel, $\tau_{\text {RMS }}$, is derived from the multipath intensity profile $\psi_{D e}(\tau)$ which represents the average power of the channel output as a function of delay time (Paulraj et al., 2003; Stüber, 1996).

$\tau_{\mathrm{RMS}}=\sqrt{\frac{\int_{0}^{\tau_{\max }}(\tau-\bar{\tau})^{2} \psi_{D e}(\tau) d \tau}{\int_{0}^{\tau_{\max }} \psi_{D e}(\tau) d \tau} .}$

$\tau_{\max }$ is the maximum delay spread while $\bar{\tau}$ is the average delay spread given by

$\bar{\tau}=\frac{\int_{0}^{\tau_{\max }} \tau \psi_{D e}(\tau) d \tau}{\int_{0}^{\tau_{\max }} \psi_{D e}(\tau) d \tau}$.

The coherence bandwidth $(\Delta f)_{\mathrm{c}}$ corresponds to the frequency lag in which the channel's autocorrelation function reduces to 0.7 (Paulraj et al., 2003). As previously described, the channel representations in the four domains are coupled through Fourier transforms. For this reasons $(\Delta f)_{\mathrm{c}}$ and $\tau_{\mathrm{RMS}}$ are connected as well. In fact the coherence bandwidth is inversely proportional to the RMS delay spread so that

$(\Delta f)_{\mathrm{c}}=\frac{\text { const }_{1}}{\tau_{\mathrm{RMS}}}$.

The same concepts are applied on the $\left\{f_{D}, t\right\}$ pair. The RMS Doppler spread, $f_{\mathrm{D}, \mathrm{RMS}}$, is derived in a similar way from $\psi_{D o}$, the Doppler power spectrum, which corresponds to the average power as a function of Doppler frequency $f_{D}$.

$$
\begin{aligned}
f_{\mathrm{D}, \mathrm{RMS}} & =\sqrt{\frac{\int_{\mathcal{F}}\left(f_{\mathrm{D}}-\bar{f}_{\mathrm{D}}\right)^{2} \psi_{D o}\left(f_{\mathrm{D}}\right) d f_{\mathrm{D}}}{\int_{\mathcal{F}} \psi_{D o}\left(f_{\mathrm{D}}\right) d f_{\mathrm{D}}}} \\
\bar{f}_{\mathrm{D}} & =\frac{\int_{\mathcal{F}} f_{\mathrm{D}} \psi_{D o}\left(f_{\mathrm{D}}\right) d f_{\mathrm{D}}}{\int_{\mathcal{F}} \psi_{D o}\left(f_{\mathrm{D}}\right) d f_{\mathrm{D}}}
\end{aligned}
$$

The integration domain, $\mathcal{F}$, corresponds to the Doppler frequency interval where the Doppler power spectrum is non zero. The coherence time $(\Delta t)_{\mathrm{c}}$ is calculated in the same way as the coherence bandwidth. It corresponds to the time lag at which the autocorrelation function drops to 0.7 (Paulraj et al., 2003). $(\Delta t)_{\mathrm{c}}$ indicates how time variant the channel is and similarly to the $\{f, \tau\}$ pair

$(\Delta t)_{\mathrm{c}}=\frac{\text { const }_{2}}{f_{\mathrm{D}, \mathrm{RMS}}}$

Figure 4 shows typical trends for $\tau_{\mathrm{RMS}}, f_{\mathrm{D}, \mathrm{RMS}},(\Delta f)_{\mathrm{c}}$, and $(\Delta t)_{\mathrm{c}}$ for different $K$-factors. Similar plots can be observed for both models $\mathcal{M}_{\alpha}$ and $\mathcal{M}_{\beta}$ for almost any cluster size and number of scatterers. As $K$ grows $\tau_{\mathrm{RMS}}$ decreases, meaning that the channel becomes rapidly less frequency selective. The same observation can be derived from the trend of $(\Delta f)_{\mathrm{c}} \cdot f_{\mathrm{D}, \mathrm{RMS}}$ and $(\Delta t)_{\mathrm{c}}$ show the same trends. For bigger $K$ 's the channel becomes increasingly less time variant. The 

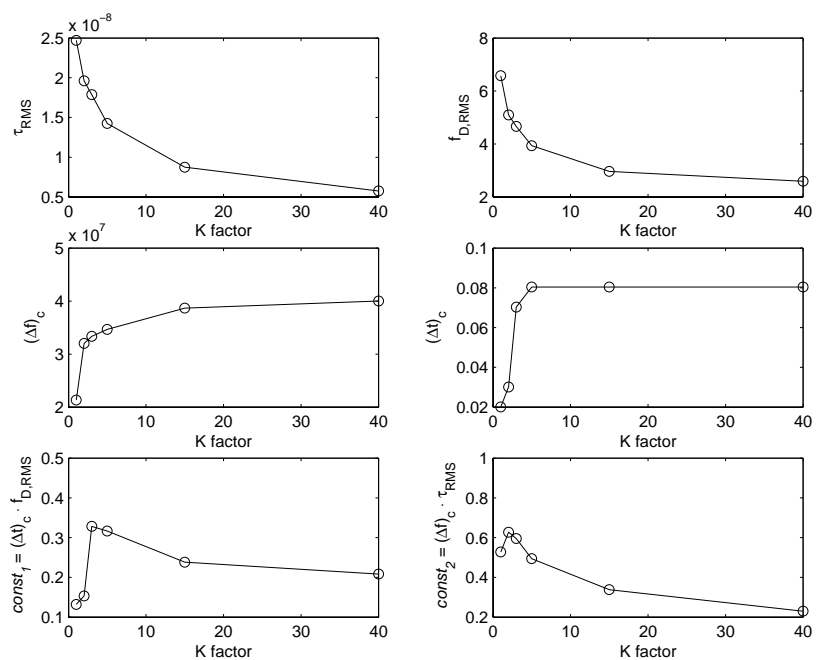

Fig. 4. $\tau_{\mathrm{RMS}}, f_{\mathrm{D}, \mathrm{RMS}},(\Delta f)_{\mathrm{c}}$ and $(\Delta t)_{\mathrm{c}}$ plotted for different $K$ factors.
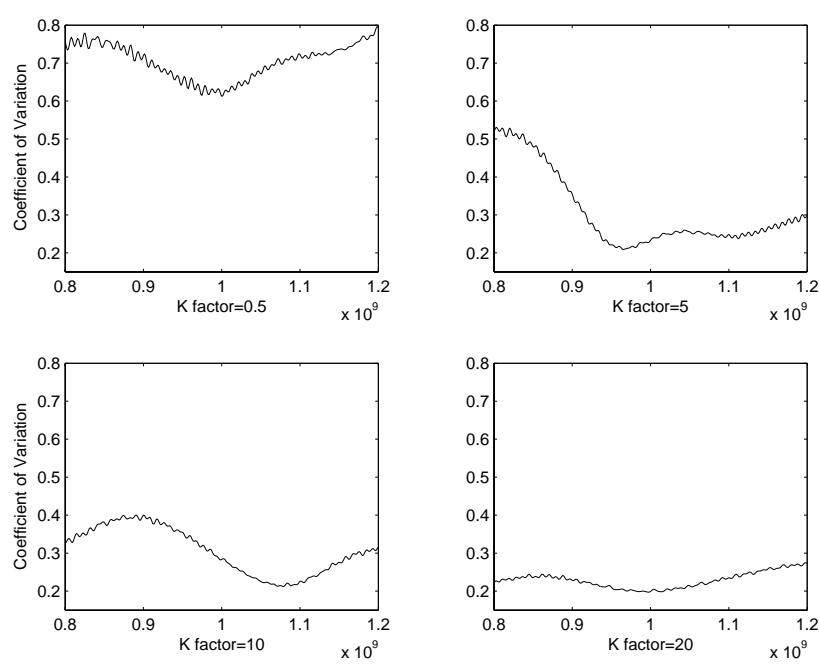

Fig. 5. The coefficient of variation $\xi$ plotted against frequency $f$ for different $K$-factors.

two constants const $_{1}$ and const $_{2}$ are plotted as well to show that the inverse proportionality expressed in Eqs. (7) and (9) is a good approximation.

Another interesting measure of the variation of the channel is to look at the fluctuation of $\|\boldsymbol{H}\|_{F}^{2}$. A good index is the so called coefficient of variation (Nabar, 2003) which is commonly used as a quantitative measure of the spread (fluctuation) of a random variable. The coefficient of variation $\xi$ is defined as:

$\xi=\frac{\sqrt{E\left\{\left(\|\boldsymbol{H}\|_{F}^{2}\right)^{2}\right\}-\left(E\left\{\|\boldsymbol{H}\|_{F}^{2}\right\}\right)^{2}}}{E\left\{\|\boldsymbol{H}\|_{F}^{2}\right\}}$.

We calculated $\xi$ for all frequency bins to discover that for growing $K$ 's the coefficient of variation becomes increasingly smaller (Fig. 5). This is actually expected if we associate
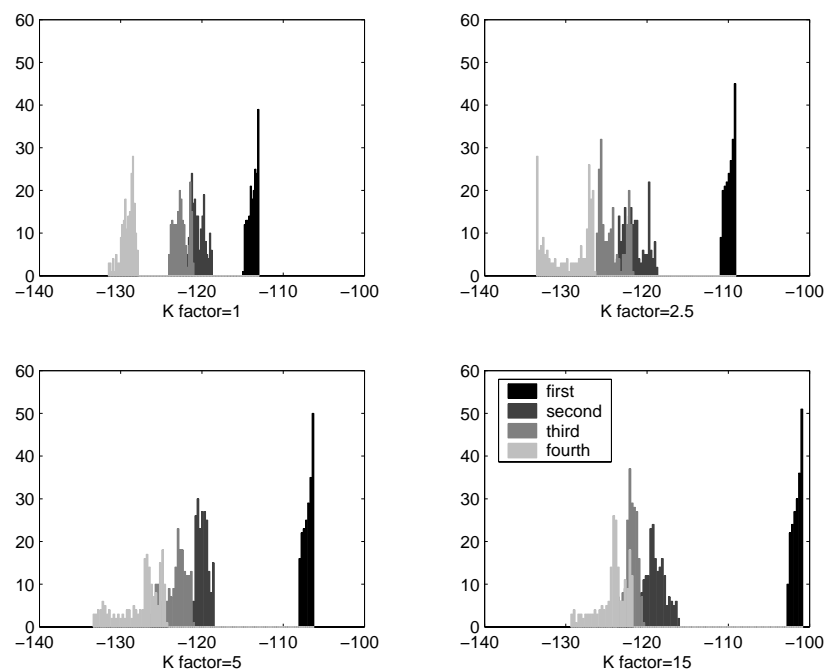

Fig. 6. Eigenvalue distributions in $\mathrm{dB}$ as $K$ grows for $\mathcal{M}_{\alpha}$.

$\xi$ with the effective diversity order, as described in (Nabar, 2003).

In fact the effective diversity order, $N_{d i v}$, can be written as:

$N_{d i v}=\frac{1}{\xi^{2}}$,

and it approaches infinity for a channel with only the LOS component (AWGN channel) while $N_{d i v}=M_{R} \cdot M_{T}$ for Rayleigh channels. The trend in $\xi$ and consequently in $N_{\text {div }}$ can be observed for both models proposed and independently from other parameters.

Another interesting analysis can be performed on the statistics of the eigenvalues. Figure 6 shows their distributions for different $K$-factors for the model $\mathcal{M}_{\alpha}$. Eigenvalue decompositions have been applied on the spatial correlation matrices at the receiver $\boldsymbol{R}_{R}$ calculated for each time snapshot. Then we generated the histograms to derive the amplitudes distribution on a $\mathrm{dB}$ scale. The spatial correlation matrix $\boldsymbol{R}_{R}$ at the receiver for the specific time $t_{o}$ is:

$\boldsymbol{R}_{R}\left(t_{o}\right)=\frac{1}{F} \sum_{i=1}^{F} \boldsymbol{H}\left(:,:, i, t_{o}\right) \cdot \boldsymbol{H}\left(:,:, i, t_{o}\right)^{H}$,

where $F$ is the total number of frequency bins, $\boldsymbol{H}^{H}$ is the Hermitian transpose of $\boldsymbol{H}$ and the channel $\boldsymbol{H}$ has dimensions $M_{R} \times M_{T} \times F \times T$. The MATLAB like notation $\boldsymbol{H}\left(:,:, i, t_{o}\right)$ denotes the two-dimensional channel matrix $\boldsymbol{H} \in \mathbb{C}^{M_{R} \times M_{T}}$ for a specific frequency $f=i$ and time $t=t_{o}$. Figure 6 shows the distributions of the eigenvalues plotted in $\mathrm{dB}$. The channels have been generated without noise and without any normalization, i.e. the absolute amplitudes of the eigenvalues appear very low (around -120 dB). This has no physical meaning and only the relative distances between the eigenvalues should be taken into account. As the $K$-factor grows, the Line Of Sight becomes increasingly stronger and the channel displays low rank traits. In other words the strongest eigenvalue separates itself from the weaker ones as $K$ increases. 

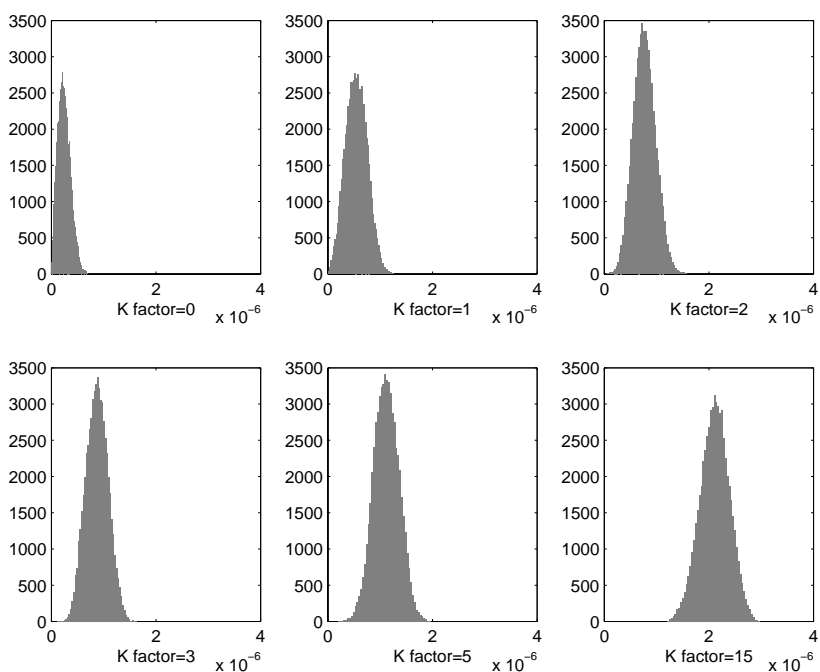

Fig. 7. Amplitude distributions of channels considering all frequencies and time snapshots for different Rician $K$-factors for $\mathcal{M}_{\alpha}$.

A higher $K$-factor inhibits the effects of the fast-fading. This can be easily seen by plotting the distributions of the amplitudes of the channel considering all frequencies and time snapshots (Fig. 7).

\subsection{Cluster size and number of scatterers}

While there exists a strong relationship between the different channel statistics and the Rician $K$-factor, there is no strict relation with the parameters which characterize a scattering cluster, namely the number of scatterers, the cluster size and the type of distribution of the scatterers inside the cluster. In the first simulations we investigated whether the geometrical distribution affected the channel. We generated clusters with scatterers distributed uniformly on a sphere, on a circle on the horizontal plane (as seen in Figs. 2 and 3), and on the surface of a cylinder or a sphere. All channels showed the same trends and for this reason we continued with more detailed investigations fixing the distribution of the scatterers on a circle. We generated models characterized by cluster sizes with diameters ranging from $\frac{\lambda}{2}$ to $20 \lambda$ and with numbers of scatterers ranging from 1 up to 100 reflection points per cluster.

We then investigated trends in the different channel features fixing all parameters as either the cluster size or the number of scatterers changed. All features but the effective diversity order did not show any strong dependency even though they were all affected. For instance, increasing the size of the scattering clusters affects the frequency selectiveness of the channel, even though there is neither a direct nor an inverse proportionality between the two. Figure 8 shows the effective diversity order $N_{\text {div }}$ plotted for different frequencies $f$ and for different cluster diameters expressed in $\lambda$ 's for the first model, $\mathcal{M}_{\alpha}$. Analyzing $N_{d i v}$ along different frequencies we can recognize a slow-moving trend corrupted by faster variations. For instance, in the first plot of Fig. 8
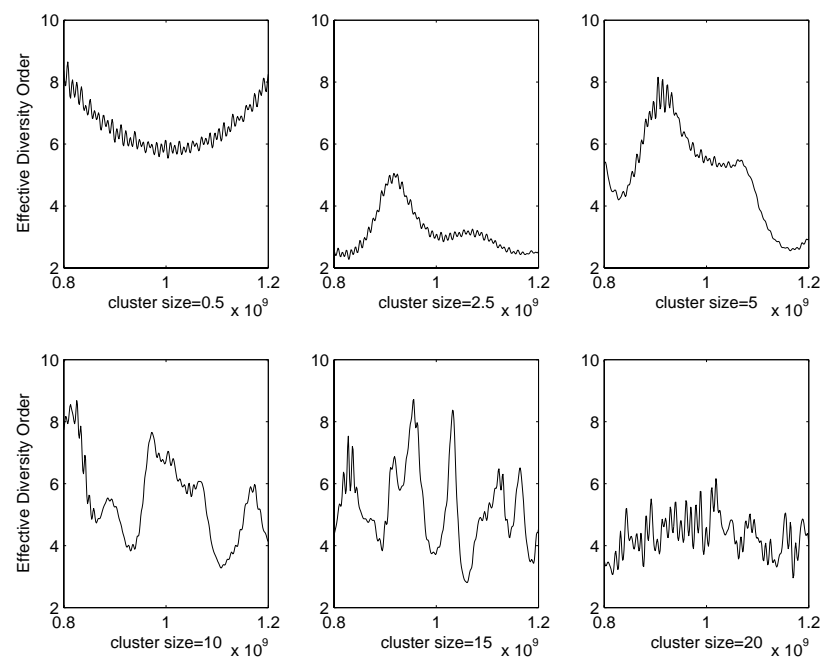

Fig. 8. The effective diversity order $N_{\text {div }}$ plotted against frequency $f$ for different cluster sizes (expressed in $\lambda$ 's) for $\mathcal{M}_{\beta}$.

(cluster size $=0.5 \lambda$ ), we see a slow moving trend which looks like the sum of a concave semicircle and a zero mean noise-like process which introduces fast variations. After many simulations we observed that the magnitude of the fast moving $N_{d i v}$ changed at every realization while the variance of the slow moving $N_{d i v}$ changed with the cluster diameter with an undoubtedly direct proportionality. In other words, for bigger cluster sizes, $N_{d i v}$ changes more rapidly along frequency while this does not necessarily correspond to higher frequency selectiveness nor to greater time variance. The number of scatterers affects the channel in a similar way but with a much weaker dependency.

\section{A comparison with measurements}

In order to assess the results derived in Sect. 3 with an even more realistic scenario we modeled the surroundings of a measurement campaign undertaken at TU Ilmenau. Additionally the simulation allowed us to investigate the importance of other critical parameters of the model: the positions of the scatterers and the modelling of the reflection coefficients. The first step was to determine whether a rough positioning of the scatterers was enough to recreate the main features which characterize the measured channel. To this end, we chose, among the many measurements gathered, a channel with a strong LOS and characterized by a simple geometry so that the position of the scatterers could easily be estimated. Figure 9 shows the bird's eye view of the reconstructed scenario. The receiver was a $4 \times 8$ polarization sensitive patch-array URA (Uniform Rectangular Antenna) and it was positioned by a window on the second floor of a building. The transmitter was a single $2 \mathrm{GHz}$ omnidirectional antenna mounted on a wheeled cart pulled by hand up a road in the direction of a second building (Fig. 10). The transmitter path is represented by a continuous line in Fig. 9 . 


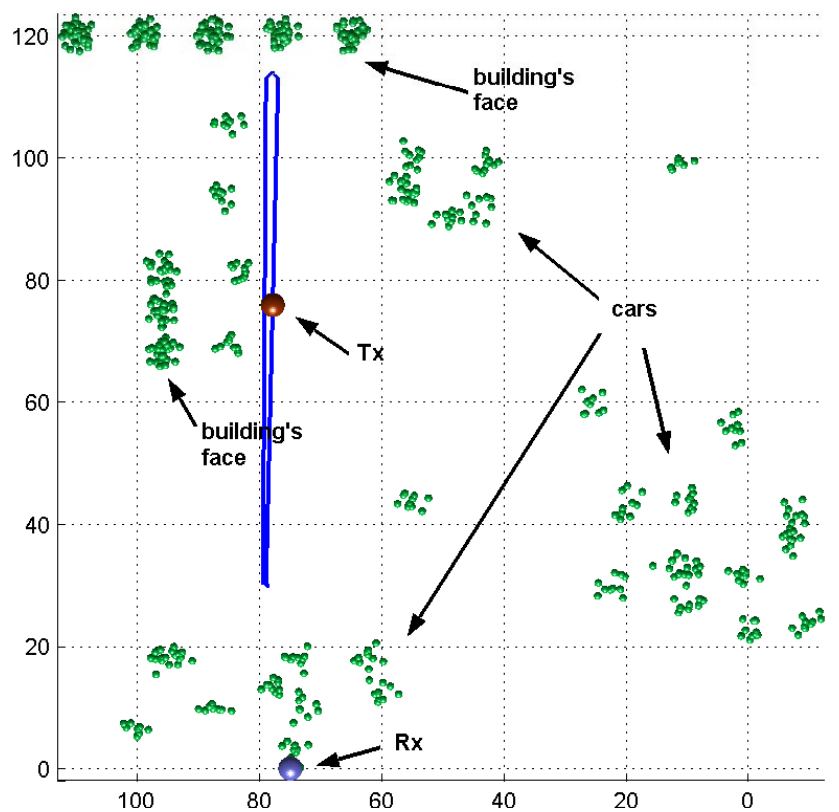

Fig. 9. Bird's eye view of the synthetic reconstruction of the scenario where the measurements were taken.

In the proximity of the building the cart was turned around and pulled in the opposite direction (towards the receiver) approximately on the same path. The whole measurement took approximately $1 \mathrm{~min}$ at a speed of $3 \mathrm{~m} / \mathrm{s}$. The measurement device employed was the broadband real-time channel sounder RUSK MIMO from MEDAV (Thomä et al., 2001). The following parameters characterize the measurements:

- A bandwidth $B$ of $120 \mathrm{MHz}$ at a center frequency of $1.95 \mathrm{GHz}$ sampled with $\Delta f=300 \mathrm{kHz}$ for a total of 385 samples.

- A total of 6313 time snapshots were taken, one every $9.2 \mathrm{~ms}$ for a total of $58 \mathrm{~s}$.

The same parameters were chosen for the synthetic IlmProp model so that the two data sets had same dimensions and sampling grids. However, in order to compare the measurements with the synthetic channels, adding the noise and performing a proper normalization must be taken into account.

The need for a normalization is due to the fact that the absolute powers sent by the antennas have not been considered. Only the relative difference in power between the LOS and the scattering components have been modeled through the Rician $K$-factor which can be set at will. This simplified the modelling effort even though the results need postprocessing in order to be finally compared with the measurements. The normalization is performed in such a way that the total power received for the synthetic channel matches the one received in the measurements. Clearly both data sets must have the same number of samples, dimensions and more in general, the same sampling grids. If $\boldsymbol{H}_{M}$ denotes the channel for the measurements and $\boldsymbol{H}_{I}$ the one generated with

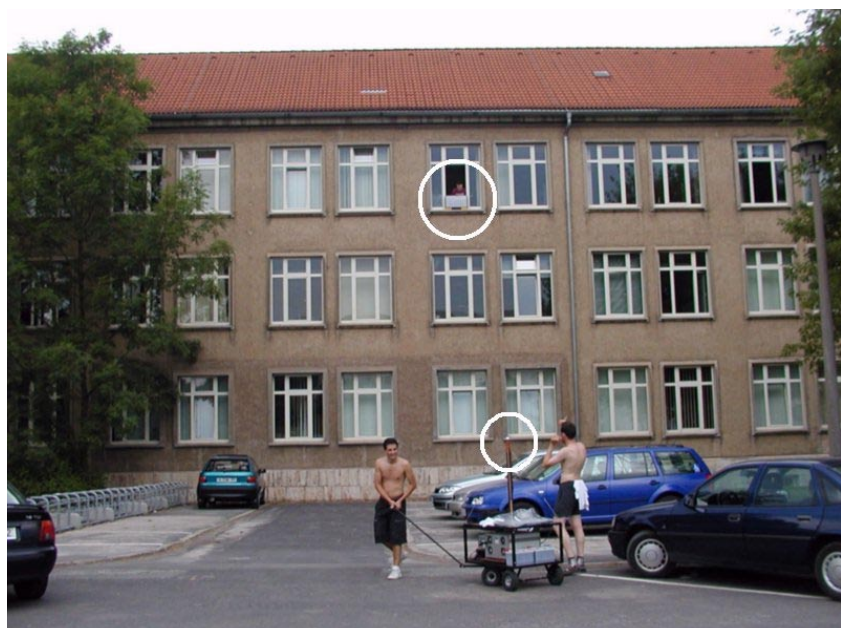

Fig. 10. The receiver (top circle) and the transmitting antenna (bottom circle) mounted on a cart.

the IlmProp then the whole channel matrix must be scaled so that

$\sum_{\Theta}\left\|\boldsymbol{H}_{M}\right\|^{2}=\sum_{\Theta}\left\|\boldsymbol{H}_{I}\right\|^{2}$

where $\Theta$ spans the complete domain of all dimensions. In our simulations $\boldsymbol{H}_{I}$ has been generated without noise. The normalization and the adding of the noise must then be done concurrently. First we have to estimate the power of the noise from the measurements. We assume constant AWGN (Additive White Gaussian Noise) over all dimensions (delay time, time, antennas, etc.). An effective way to measure the noise floor in the measurements is to observe the channel matrix in the time and delay time domain. If the path lengths are short enough, so that the last echoes extinguish before the maximum delay resolvable, we have a measurement of the noise without signal. If the number of samples is sufficient we can use this data to estimate the power of the noise. The noisy synthetic channel will then be

$\boldsymbol{H}_{I, \text { noisy }}=\boldsymbol{H}_{I, \text { noiseless }}+\boldsymbol{H}_{w}$,

where $\boldsymbol{H}_{w}$ is a matrix whose elements are ZMCSCG (Zero Mean Circular Symmetric Complex Gaussian) random numbers with variance $\sigma^{2}$ (Paulraj et al., 2003).

Equation (13) then becomes:

$\sum_{\Theta}\left\|\boldsymbol{H}_{M}\right\|^{2}-\gamma \cdot \sigma^{2}=\sum_{\Theta}\left\|\boldsymbol{H}_{I}\right\|^{2}$

where $\gamma$ is the total number of samples present in $\boldsymbol{H}$, i.e. $\gamma=M_{R} \cdot M_{T} \cdot T \cdot F$, where $T$ and $F$ are the number of time snapshots and frequency bins, respectively.

The geometry has been roughly reconstructed with the help of blueprints of the area and through visual inspection. Cars, buildings, light poles, and trash bins have been identified as the probable sources of scattering.

Figure 11 shows the channel impulse responses in time $t$ and delay time $\tau$. The channel has been gathered originally in the $\{t, f\}$ domain; the CIRs in delay time have been computed via the Fourier transform described in Eq. (1). The 


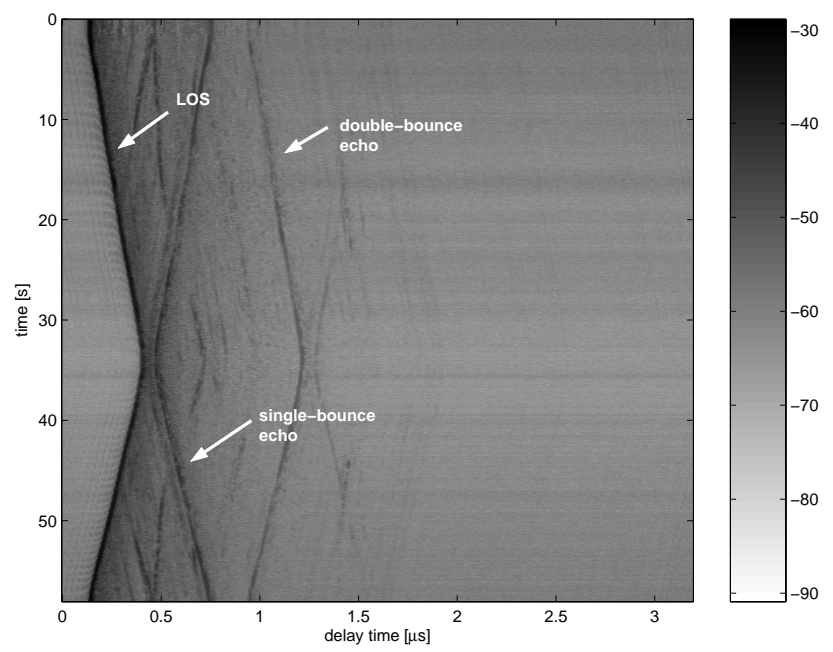

Fig. 11. Channel Impulse Responses from the measurements in time $t$ and delay time $\tau$. The Line Of Sight LOS and echos are clearly visible. The grayscale represents amplitudes in a dB scale

LOS is clearly visible and as expected its delay lag grows as the transmitter moves further away from the receiver. When the cart is turned around and pulled back towards the receiver, the arrival delay time decreases. Many echoes are visible as well. Note that as the transmitter moves most echoes arrive earlier, meaning that their path length becomes smaller. When the curve corresponding to one echo changes in time with the same slope characterizing the Line Of Sight, then we can deduce that the path length of the echo changes with the same rate of the path length of the LOS. The strong echo labeled double-bounce echo has the same trend as the LOS just delayed by approximately $0.8 \mu \mathrm{s}$, which corresponds to $240 \mathrm{~m}$, the distance between the top and bottom buildings doubled. For this reason it is very likely that this arrival corresponds to the signal reflected by the bottom building first, and then by the top building, thus a doble-bounce echo. Figure 12 shows the channel generated by our model. The trend of the LOS is extremely well reproduced. This was, however, expected since the LOS is the only component easily predictable once the position of the transmitter in known. Most of the scattering contributions can be observed in the synthetic data set as well, with the exception of the double-bounce paths which have been omitted. Analyzing the two data sets in the $\{t, \tau\}$ domain suggests improvements in the modelling approach: in the measurements we can observe that certain echo traces are stronger than others. In the modeled channel this does not happen. This is a result of the modelling of the reflection coefficients. In fact while their phase has been generated uniformly distributed in the $[0,2 \pi]$ range, the amplitude has been taken equal for all. The comparison suggests that the reflection coefficients need a more sophisticated modelling. Furthermore, a closer look on the measurements reveals that some echoes exist only in certain time periods. This suggests that the amplitude of the reflection coefficients (perhaps their phases too) should be

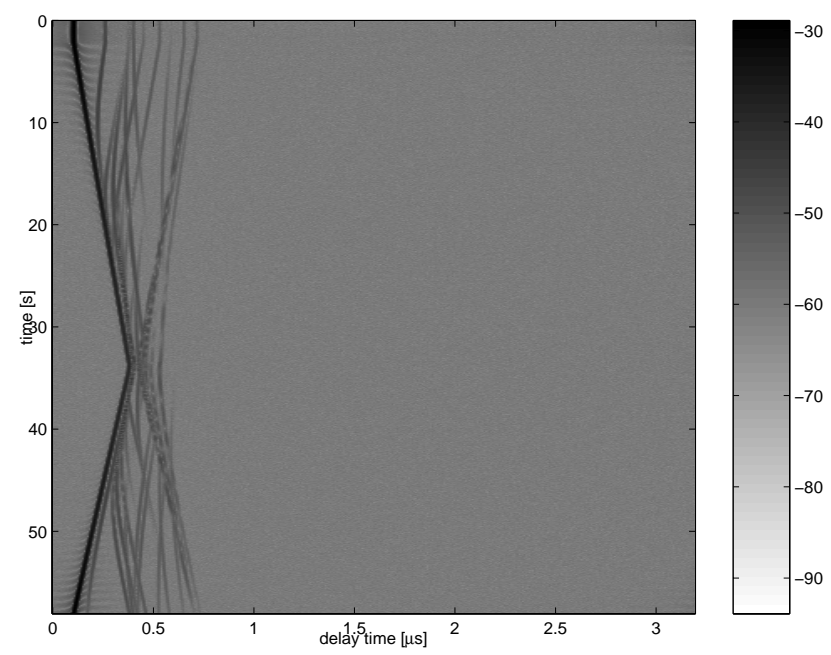

Fig. 12. The synthetically generated Channel Impulse Responses in time $t$ and delay time $\tau$.
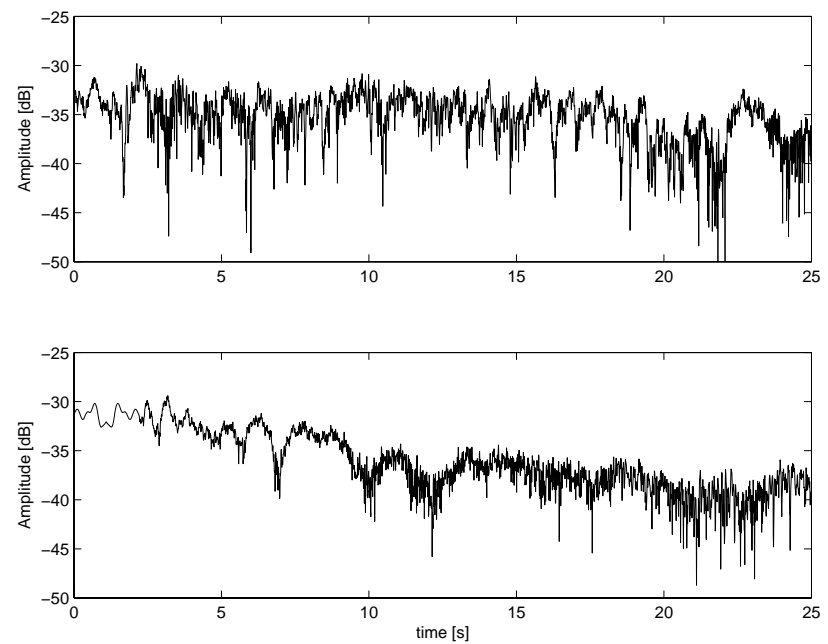

Fig. 13. Amplitudes of the CIRs for a specific frequency bin plotted in $\mathrm{dB}$ against time for the measurements (top) and the IlmProp model (bottom).

dependent on the Direction of Arrival (DoA) and Direction of Departure (DoD). Figure 13 shows the fluctuations of the amplitudes of the channel for a specific frequency $f_{o}$ plotted against time for the first $25 \mathrm{~s}$. Fast-fading characterizes both signals even though the measurements appear somehow more variant. In order to assess this feature quantitatively we compute the multipath intensity profile $\psi_{D e}(\tau)$, the Doppler power spectrum $\psi_{D o}\left(f_{D}\right)$ and the autocorrelation functions in frequency $\operatorname{acf}(\Delta f)$ and time $\operatorname{acf}(\Delta t)$ for both data sets. From their analysis we can obtain the RMS delay spread $\tau_{\mathrm{RMS}}$, the coherence bandwidth $(\Delta f)_{\mathrm{c}}$, the RMS Doppler spread $f_{\mathrm{D}, \mathrm{RMS}}$ and the coherence time $(\Delta t)_{\mathrm{c}}$ (Figs. 14 and 15). The RMS delay spread shows that the energy is similarly distributed in delay time $\tau$. In the measurements two 

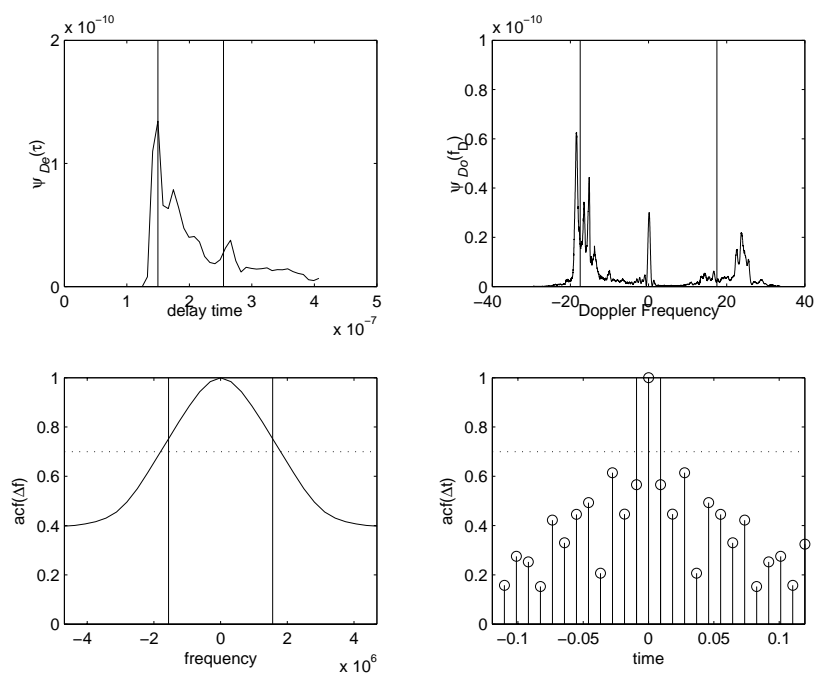

Fig. 14. The multipath intensity profile $\psi_{D e}(\tau)$, Doppler power spectrum $\psi_{D o}\left(f_{D}\right)$ and the autocorrelation functions in frequency $\operatorname{acf}(\Delta f)$ and time $\operatorname{acf}(\Delta t)$ for the measurements

more spikes can be observed. This is due to the fact that two specific echoes have a significantly stronger magnitude compared to other echoes. The RMS delay spread $\tau_{\text {RMS }}$ is anyhow very similar suggesting that the two channels will have a similar frequency selectiveness. The RMS delay spread corresponds to the distance between the two vertical lines plotted in Figs. 14 and 15. From the autocorrelation function in the frequency domain $\operatorname{acf}(\Delta f)$ we can derive the coherence bandwidth $(\Delta f)_{\mathrm{c}}$ which confirms that the two channels have approximately the same coherence bandwidth. In the $\left\{t, f_{D}\right\}$ pair the two channels show more differences. While the Doppler power spectrum $\psi_{D o}\left(f_{D}\right)$ has very similar trends, the autocorrelation function in the time domain shows that the measured channel is much more time variant than the synthetic one. Furthermore this analysis proves that Eq. (9) only represents a good empirical law, valid in most but not all cases. In Fig. 13 we can in fact recognize that the measurements have a faster moving small-fading effect, especially during the first $10 \mathrm{~s}$. We then investigated how we could generate more time variance changing only positions, the size of the scattering clusters, and the number of scatterers per cluster, discovering that this was not possible. Our simulations showed that in order to achieve higher order of time variance it is absolutely necessary to model time variant reflection coefficients or to introduce moving scatterers along the time dimension.

\section{A deeper look at the phase response}

Looking more closely at the phase of the received signal leads to interesting comparisons and remarks. Figure 16 shows how the phase of the received signal (in its complex base-band representation) is constructed in a flat-fading frequency selective channel. The resulting phase $\varphi_{R}$ will be
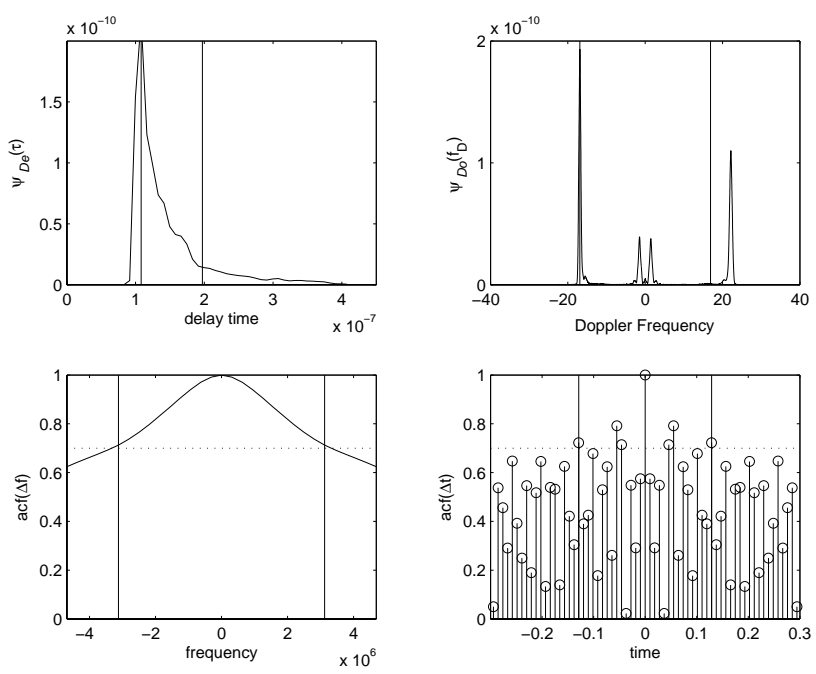

Fig. 15. The multipath intensity profile $\psi_{D e}(\tau)$, Doppler power spectrum $\psi_{D o}\left(f_{D}\right)$ and the autocorrelation functions in frequency $\operatorname{acf}(\Delta f)$ and time $\operatorname{acf}(\Delta t)$ for the IlmProp model

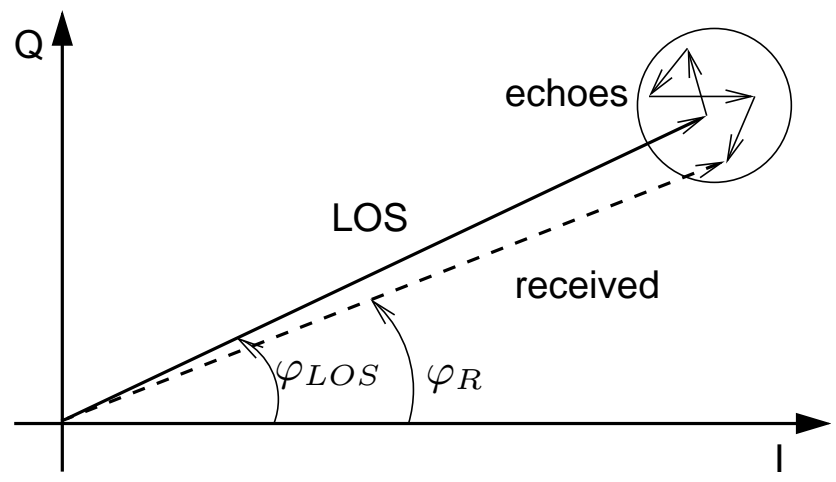

Fig. 16. When the amplitude of the echoes is small the phase of the received signal $\varphi_{R}$ is a good approximation of the phase of the LOS component only $\varphi_{L} O S$.

the sum of the phase introduced by the LOS $\varphi_{L O S}$ plus the additional phase introduced by the echoes. The smaller the amplitude of the echoes is compared to the LOS component, the smaller will be the difference $\varphi_{R}-\varphi_{L O S}$. As the radial distance between transmitter and receiver changes, the LOS component, $\varphi_{L O S}$, will change accordingly. If the movement is slow enough and the phase-noise introduced by the echoes is negligible, it is possible to derive the displacement from the phase trend of the received signal. In fact, a movement of $\lambda$ in the radial direction corresponds to a full rotation of $2 \pi$ radians in the phase. If this wavelength has been sampled with more than two samples per wavelength, thus fulfilling Shannon's theorem, the phase can be efficiently 'unwrapped', or in other words, absolute jumps greater than $\pi$ are converted to their $2 \pi$ complement. The spatial displacement can be then derived from the phase simply multiplying by the factor: $\frac{\lambda}{2 \pi}$. Figure 17 shows the unwrapped phase for every frequency bin derived directly from the measured 


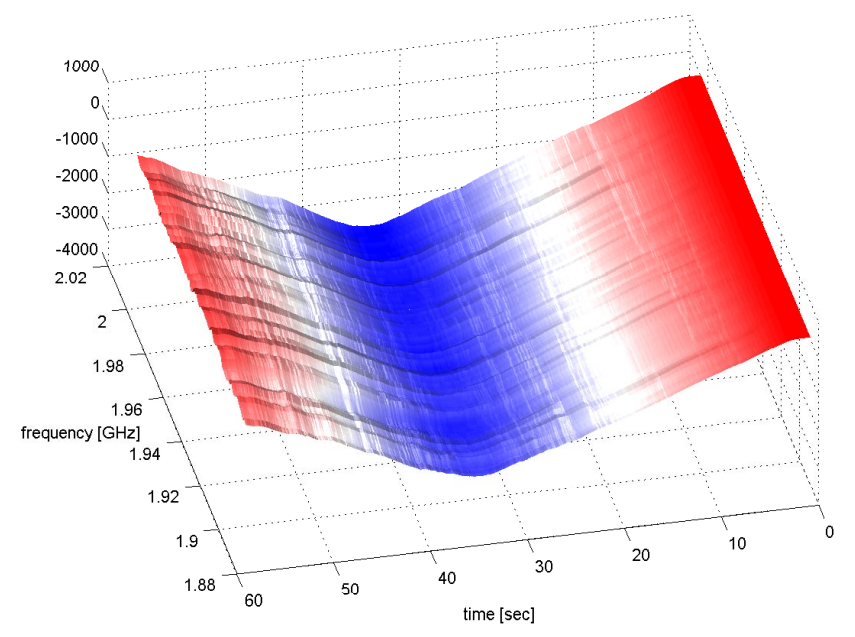

Fig. 17. Unwrapped phase trend derived from measurements plotted in radians against frequency $f$ and time $t$.

channel analyzed in Sect. 4. Even though a different fluctuation perturbs each frequency bin, the overall trend is constant and shows a descending phase for half of the time and a symmetrical ascending phase for the other half, in accordance with the experiment's geometry. The different slope is due to the different velocity. In fact the transmitter moved faster in the second half of the experiment. If the channel were flat-fading we would see the slow moving LOS component only, absolutely equal for every frequency. It is interesting to compare the overall phase trend with the LOS component only. In case of the synthetic channel this is very easy to obtain. More complicated is the case of the measurements. Once the channel has been transformed in the $\{t, \tau\}$ domain, it is possible to identify the samples in delay time $\tau$ with highest energy for every time snapshot. The changing phase of these samples against time approximates the phase trend introduced by the LOS component only. The comparison of the unwrapped LOS phase with the overall phase is shown in Fig. 18. The model and the measurements show very similar traits. The upper curves show the phase trend for one frequency bin derived directly from the unwrapping of the phase. The lower curves are derived, respectively, with the LOS approximation for the measurements, and from a direct calculation of the displacement for the model. In fact, for the synthetic data, the exact path of the transmitter is known. From these coordinates it is possible to derive the radial displacement and consequentially the phase. The phase trends match for the first $10-15 \mathrm{~s}$, while they start to diverge significantly around $25 \mathrm{~s}$. This can be simply explained considering that the Rician $K$-factor drops in the middle part of the experiment. When the amplitude of the echoes is comparable with the LOS, then the received vector will have a phase uniformly distributed distributed in the interval $[0,2 \pi]$. The unwrapper will not find any trend and will jump from ascending to descending phases randomly. The resulting unwrapped signal will have a slope close to 0 , that is horizontal. For this reason, as we pass from a higher $K$-factor to a smaller one we

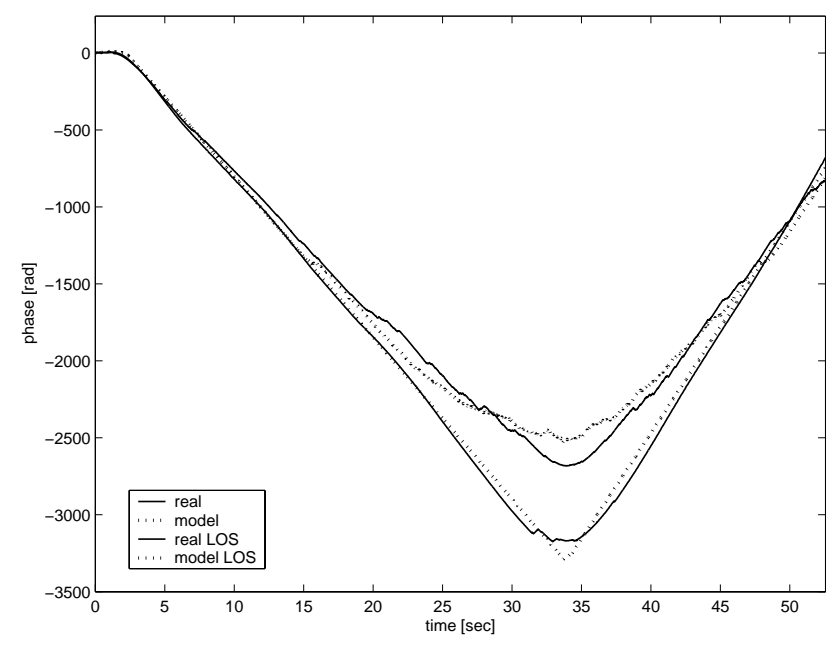

Fig. 18. Phase comparison between the measurements and its synthetic reconstruction. The upper curves are obtained simply unwrapping the signal received at one specific frequency. The lower curves represent the LOS component only.

find increasingly less steep slopes. In other words the phasenoise introduced by the echoes is uniformly distributed, but due to the non-linearity of the unwrapper, the resulting error leads always to more moderate slopes.

\section{Conclusions}

Our simulations show that for certain modelling applications a simple geometry-based single-bounce model can mimic most channel features with sufficient precision, among which the frequency selectiveness and the time variance are the most characterizing. A rough positioning of the scattering clusters allows us to achieve the desired frequency selectiveness as well as other specific traits of the channel statistics by tuning three basic parameters, i.e, the size of the scattering clusters, the number of scattering coefficients, and the Rician $K$-factor. To obtain higher levels of time variance and more realistic synthetic channels, moving scatterers and time dependent reflection coefficients must be modeled as well.

Acknowledgements. The authors gratefully acknowledge the support of MEDAV (www.channelsounder.de) and of the Electronic Measurements Engineering Laboratory at Ilmenau University of Technology in performing the channel measurements.

\section{References}

Bello, P.: Characterization of randomly time-variant linear channels, IEEE Trans. Comm. Syst., 11, 360-393, 1963.

Correia, L. M.: Wireless Flexible Personalised Communications, Wiley, 2001.

Nabar, R. U.: Performance Analysis and Transmit Optimization for General MIMO Channels, Doctoral Dissertation, Stanford, 2003. 
Paulraj, A., Nabar, R., and Gore, D.: Introduction to space-time wireless communications, Cambridge University Press, Cambridge, 2003.

Stüber, G.: Principles of Mobile Communication, Kluwer, Norwell, MA, 1996.
Thomä, R., Hampicke, D., Richter, A., Sommerkorn, G., and Trautwein, U.: MIMO vector channel sounder measurement for smart antenna system evaluation, European Transaction on Telecommunications, Special issue on smart antennas, 12, 2001. Van Trees, H. L.: Optimum Array Processing: Part IV of Detection, Estimation, and Modulation Theory, John Wiley \& Sons Inc, New York, 2002. 